\title{
Development of the time domain simulation of impulsive radio signals for ARAcalTA
}

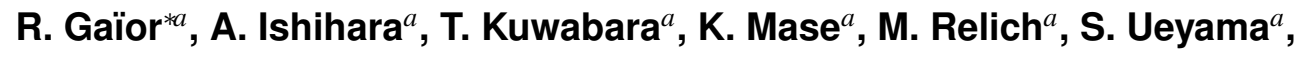

S. Yoshida ${ }^{a}$ for the ARA collaboration,

M. Fukushima ${ }^{b}$, D. Ikeda ${ }^{b}$, J. N. Matthews ${ }^{c}$, H. Sagawa ${ }^{b}$, T. Shibata ${ }^{d}$, B. K. Shin ${ }^{e}$, G. B. Thomson ${ }^{c}$.

${ }^{a}$ Department of Physics, Chiba University, Yayoi-cho 1-33, Inage-ku, Chiba 263-8522, Japan

${ }^{b}$ ICRR, University of Tokyo, 5-1-5 Kashiwanoha, Kashiwa, Chiba 277-8522, Japan

${ }^{c}$ High Energy Astrophysics Institute and Department of Physics and Astronomy, University of Utah, Salt Lake City, Utah, USA

${ }^{d}$ High Energy Accelerator Research Organization (KEK), 2-4 Shirakata-Shirane, Tokai-mura, Naka-gun, Ibaraki, 319-1195, Japan

${ }^{e}$ Department of Physics, Hanyang University, 222 Wangsimni-ro Seongdung-gu Seoul, 133-791, Korea

*E-mail: gaior@hepburn.s.chiba-u.ac.jp

The Askaryan effect is the coherent radio emission from an electron excess in a particle cascade. ARA (Askaryan Radio Array) is a radio detector being built to observe the Askaryan radiation from the cascade in ice induced by ultra high energy neutrino $(\mathrm{E}>10 \mathrm{PeV})$ around the South Pole. In order to study further the characteristics of the coherent radiation, and also validate ARA detection system response, we set up a replica of the ARA experiment, the ARAcalTA. We used the electron linear accelerator on the Telescope Array site to shoot $40 \mathrm{MeV}$ electron bunches in an ice target. The electron excess in ice provokes a coherent radiation that is detected by the ARA sensors. Because of the impulsive nature of the expected signal, we developed a simulation chain entirely in the time domain (instead of frequency). We present the simulation combining a Geant 4 particle tracking and a particle per particle radio emission calculation. These results are in turn linked to the detector calibration and simulation to obtain the final expected waveform for ARAcalTA. We demonstrate that in absence of other background, the coherent radiation from the electron excess can be observed and characterized with ARAcalTA.

The 34th International Cosmic Ray Conference,

30 July- 6 August, 2015

The Hague, The Netherlands

\footnotetext{
*Speaker.
} 


\section{Introduction}

The nature of Ultra High Energy Cosmic Rays (UHECRs) still remains unrevealed. Beside the direct detection, another path exists to probe UHECRs. The UHE neutrinos $(\mathrm{E} \geq 10 \mathrm{PeV})$ are one such avenue and could be the key to shedding light on the composition, source and thus the production mechanism of these high energy particles.

ARA, the Askaryan Radio Array [1], is a radio detector currently being constructed $200 \mathrm{~m}$ deep in the ice of Antarctica and aims at the detection of such UHE neutrinos. The electromagnetic shower induced by an interaction of a neutrino with an ice nucleus gives rise to a negative charge excess due to Compton scatterings and positron annihilations. This excess produces in turn a coherent radio pulse of $\simeq 1 \mathrm{~ns}$, the so called Askaryan radiation. First predicted by Askaryan in 1962 [2], this phenomenon was observed in a laboratory in dense media target [3], and also in an air shower radio experiments [4].

In order to complete previous studies on Askaryan radiation $[3,5,6]$, and verify our detector understanding to an impulsive input, we set up a replica of the ARA experiment, ARAcalTA, at the Telescope Array Electron Light Source facility. We used the electron beam to produce an electromagnetic shower in a block of ice and the resulting radiation was collected with ARA antennas. The experiment was carried out in January 2015. The present contribution describes the simulation studies for ARAcalTA from the electric field production from the charge excess in ice to the detector simulation. The experimental results are presented in [7].

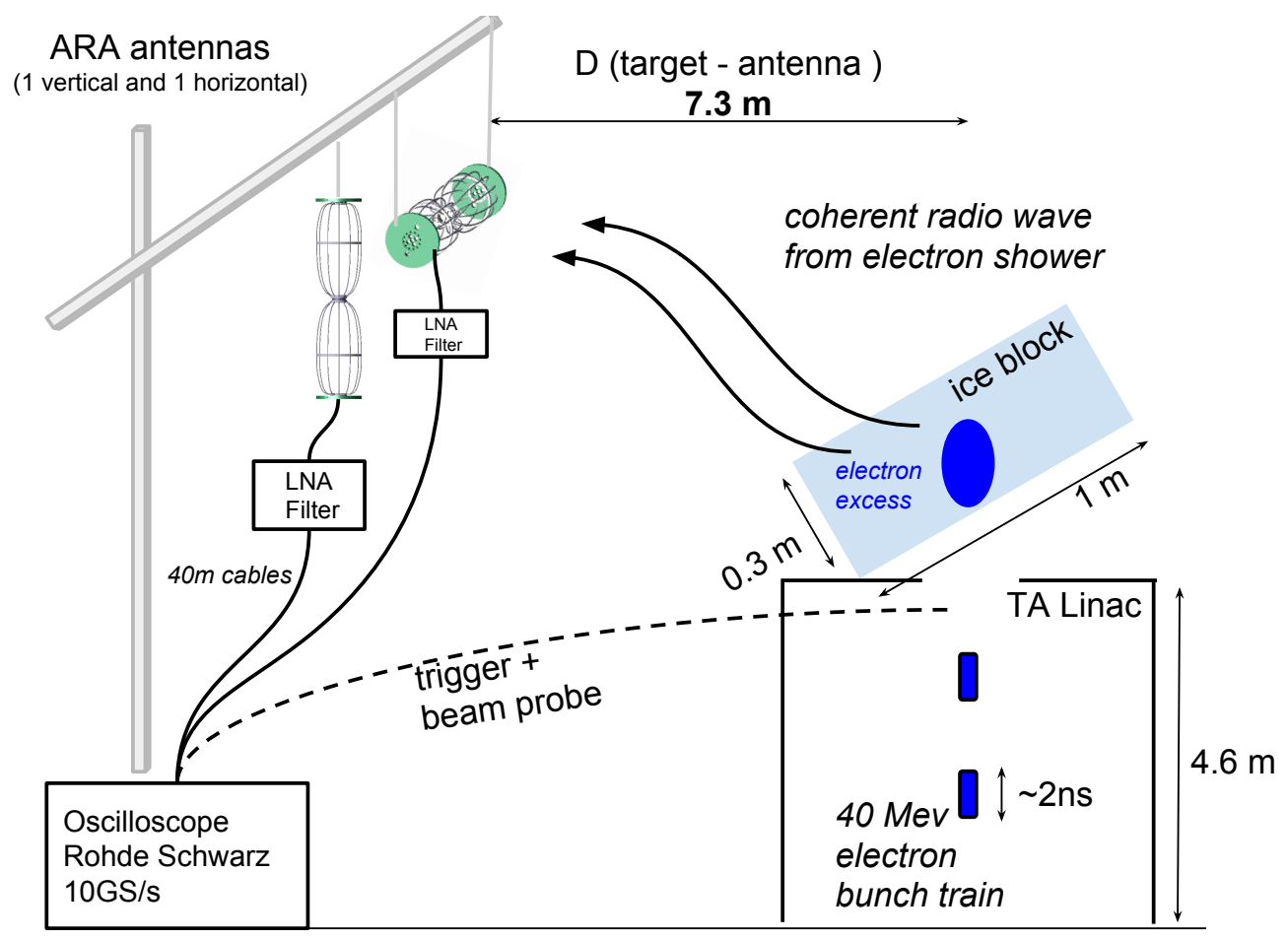

Figure 1: ARAcalTA experiment scheme. 

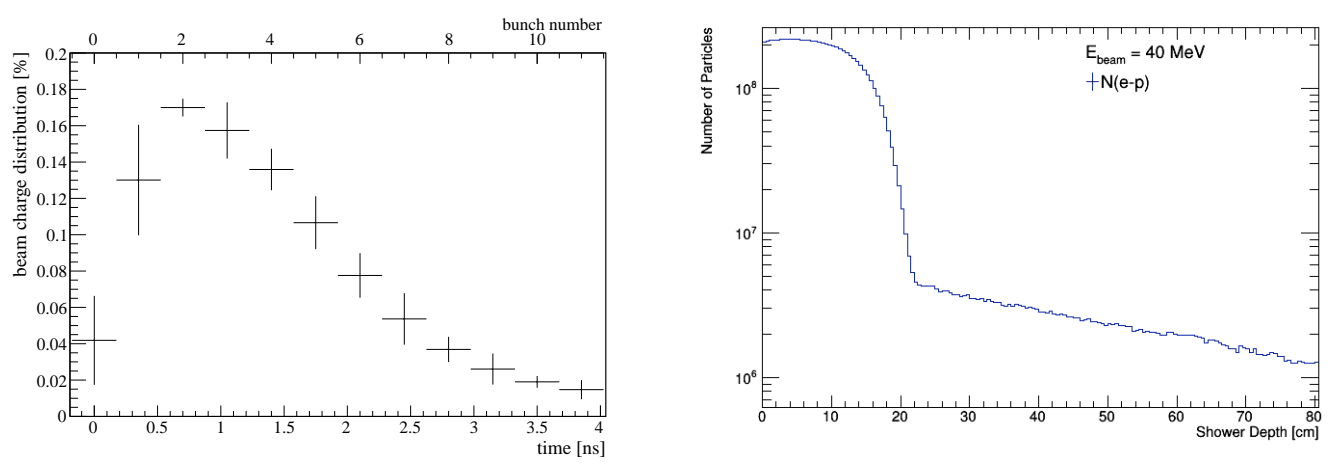

Figure 2: Left: Charge distribution in time of a bunch train measured by Faraday cup. Right: Simulated representative particle distribution in ice for $10^{9}$ primary electrons.

\section{ARAcalTA experiment}

ARAcalTA emulates the electron excess with the Electron Light Source (ELS) [8] developed for the Telescope Array experiment. The ELS is a LINAC type accelerator; it delivers a succession of accelerated electron bunches spaced by $350 \mathrm{ps}$. These bunches are grouped into bunch trains emitted at a rate of $0.5 \mathrm{~Hz}$. The duration of the bunch train is nominally more than $20 \mathrm{~ns}$, however simulation shows that a shorter bunch train induces an enhanced radio signal. Thus, an additional high voltage pulser was added for our purpose to reduce the total bunch train width to around $2 \mathrm{~ns}$ with a time profile as shown in fig. 2 (left). The electron energy is $40 \mathrm{MeV}$ and the total charge of each bunch train was measured between 10 and $50 \mathrm{pC}$. The ELS is initially designed to shoot upward in the air to measure the fluorescence light with the TA telescopes. We set up a block of ice of $1 \mathrm{~m} \times 0.3 \mathrm{~m} \times 0.3 \mathrm{~m}$ on top of the beam to produce an electron excess in it as a source of Askaryan radiation (cf experiment scheme in fig. 1). The block of ice is placed in a plastic box in which a slit was cut off to let the beam enter the ice without crossing any material before. The ELS is equipped with two types of probes to measure the beam charge: the Faraday Cup (FC) stops the beam and collects all the charge and the Wall Current Monitor (WCM) allow the bunches to pass unaffected while measuring a charge proxy. We took dedicated runs with the two probes to have precise measure of the beam profile and also to calibrate the WCM with the FC. For regular runs, only WCM output was measured, allowing us to deduce the charge event by event.

We used as a sensor the model of antenna used in ARA for the measurement of the vertical polarization. We set up two of them, spaced by $1.3 \mathrm{~m}$ and oriented orthogonally to measure simultaneously the two polarization directions (cf fig. 1). Each antenna is directly followed by a custom made band pass filter to select a quiet range of frequencies between $230 \mathrm{MHz}$ and $430 \mathrm{MHz}$ and a stage of amplification of $35 \mathrm{~dB}$. The signal is then transported with a 40 meters low attenuation cable $(3 \mathrm{~dB}$ maximum insertion loss) to the oscilloscope where it is acquired at a sampling rate of $10 \mathrm{GSa} / \mathrm{s}$.

The radiation issued from the electron beam in ice is comparable to the one provoked by the electron excess in a neutrino induced shower. In order to probe the angular distribution of the Askaryan radiation in ice, we scan the angular space by acting on two parameters: the height of the antenna was varied from $0 \mathrm{~m}$ (horizontal to beam exit) to $7 \mathrm{~m}$, we also took advantage of the refraction at the ice boundary and tilted the ice block to several angles $\left[30^{\circ} ; 45^{\circ} ; 60^{\circ}\right]$. This set of configurations 

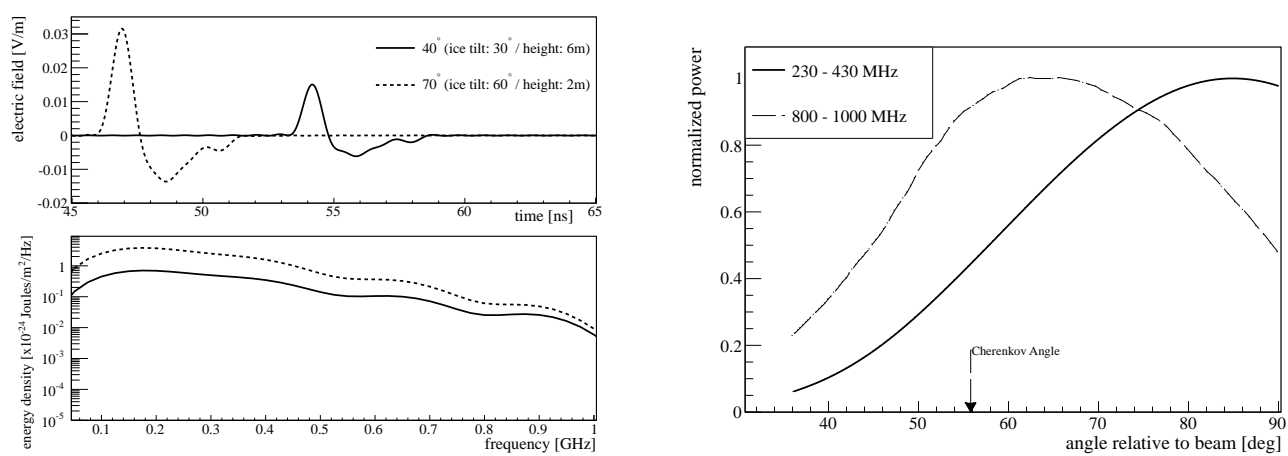

Figure 3: Left: Example of expected pulse and spectrum for angles relative to the beam of $40^{\circ}$ and $70^{\circ}$. The ice tilt and height to probe these angle are indicated in the legend. Right: Angular distribution of the power integrated over the measured frequency range [230-430MHz] (solid line) and at higher frequency (dashed line).

allowed us to cover a range from 40 to 75 degree in emission angle with respect to the beam axis.

\section{Radio signal simulation}

The simulation of electric field produced by electromagnetic shower at Ultra High Frequencies (UHF) has been developing in the recent years due to the increasing interest for radio detection of air shower or neutrino in dense media. Radio signal parameterizations exist [9] however they are tuned for high energy showers i.e. with a development length of tens of meters in ice, whereas in the case of ARAcalTA, due to the energy of the primary electrons, the shower will be contained in a few tens of $\mathrm{cm}$. Thus a dedicated simulation was implemented based on Geant4 [10] for the simulation of the particle interaction in ice and the ZHS method [11] for the radio emission simulation.

\subsection{Ice shower simulation}

For the electron injection in ice, we used as an input a time profile deduced from beam charge measurement. The charge distribution averaged and normalized is shown in fig. 2 (left), it is composed of several individual bunches inside a global envelope whose rising or falling time is of the order of $1 \mathrm{~ns}$. We also account for a lateral Gaussian spread of electrons around the beam axis when entering the ice. This spread is due to beam divergence and was estimated by ELS beam simulation to $4.5 \mathrm{~cm}$. The pulse shape and its spread around the axis are indeed important for the expected radio signal since they can induce changes in the coherence condition. A short pulse $(\leq$ $3 \mathrm{~ns}$ ) will keep the coherence in the frequency region of our interest, while a longer pulse will destroy coherence at low frequencies. A beam spread in the lateral dimension will result in a lower signal than a idealized point like beam, although the effect is small at a few hundreds of MHz. The final electron profile in ice is shown in fig. 2 (right). A small showering part can be seen in the first interactions, however because of the low primary energy the number of secondaries is rather small and the excess charge is created mainly by the primary electrons from the bunch. 


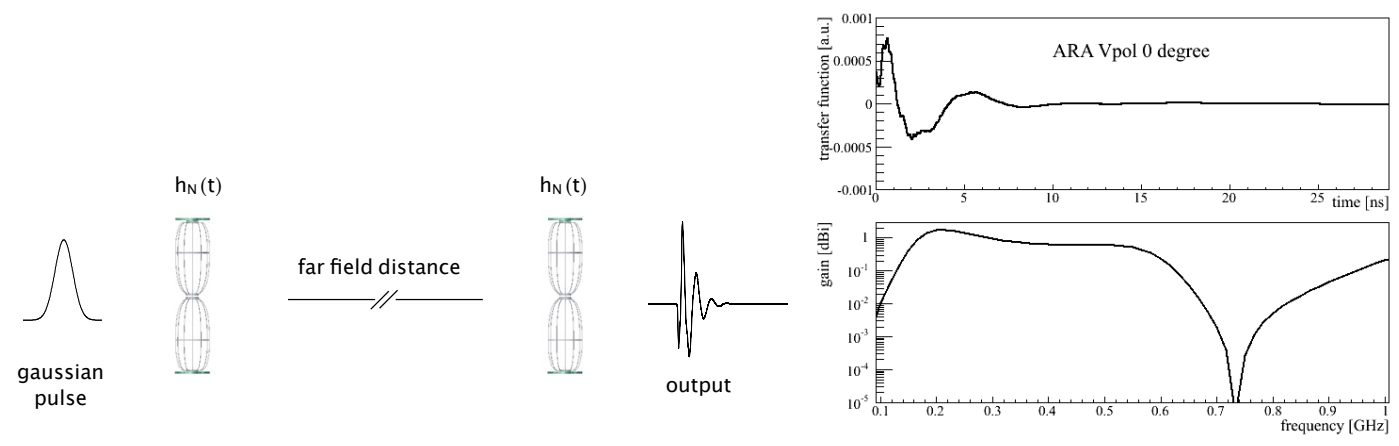

Figure 4: Left: Setup for simulation or measurement of the time domain antenna response. Right: Example of a time domain response $h_{N}(t)$ and the corresponding gain.

\subsection{Electric field simulation}

Several methods exist to compute the electric field at UHF from particle shower [12, 13, 11]. We applied the ZHS method described in [11, 14]. In this method, each physical particle track is segmented in virtual sub-tracks and the vector potential $\vec{A}_{\text {seg }}$ is computed for a given observer for each track segment according the following formula:

$$
\vec{A}_{\text {seg }}=\frac{e}{4 \pi R c} \frac{-[\hat{u} \times(\hat{u} \times \vec{\beta})]}{1-n \vec{\beta} \cdot \hat{u}}
$$

Where $R \hat{u}$ is the vector from the track segment to the observer, $R$ being its norm and $\hat{u}$ its direction, $\vec{\beta}$ is the velocity Lorentz factor and $n$ the refractive index (fixed to 1.78 in our case). For each contribution from a sub-track, the potential vector is computed at a specific detector position. The electric field is obtained by time derivation. An example of simulated electric field and its frequency spectrum is shown in fig. 3 (left) for two set of experimental configuration. The expected signal is a bipolar pulse of $5 \mathrm{~ns}$ long comparable with the type of signal expected in the ARA experiment. The simulated angular distribution shown in fig. 3 (right, solid line) exhibits a maximum around 85 degree and a wide spread around the maximum whereas in the case of a high energy shower, one expect a peaked distribution around the Cherenkov angle, i.e. 55.8 degree at these frequencies in ice. This is due to the limited effect of the Cherenkov compression because of the small dimension of the shower with respect to the wavelength. Indeed, when looking at higher frequencies (fig. 3 right, dashed line), the angular distribution tapers and converges towards the expected angle.

\section{Detector simulation and validation}

\subsection{Detector simulation}

The expected signal in ARA or ARAcalTA is a bipolar pulse of a few nanoseconds. It is then crucial to understand the response of ARA antenna for such a short input. The detector for ARAcalTA is composed of the vertical polarization antenna used in ARA, an electronics box containing a LNA and a band pass filter, and a $40 \mathrm{~m}$ low attenuation cable. For the electronic devices and cables, we measured the gain and phase with a network analyzer. We can then easily simulate their time domain response with an inverse Fourier transform. 

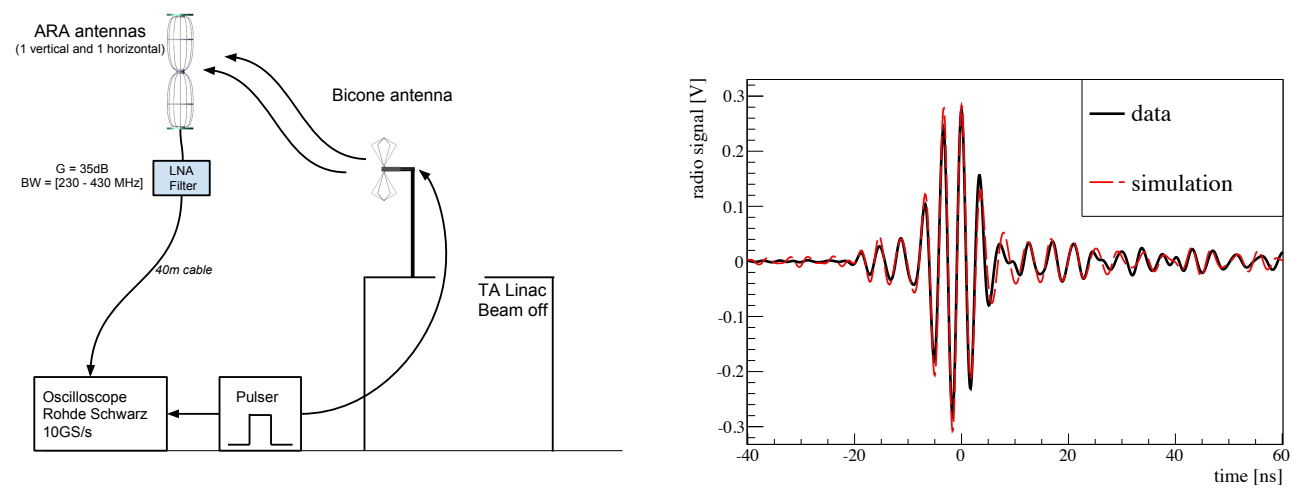

Figure 5: Left: Setup of the bicone calibration check. The emitter, the bicone is excited with a short $(\sim \mathrm{ns})$ pulser. Right: Waveform measured with ARA antenna and emitted with bicone antenna (in black), compared to the simulated waveform (in red).

The time domain response of the antenna is estimated according the method described in [15]. In this paper is defined the function $h_{N}(t)$, the time domain response of the antenna, which is the equivalent to a transfer function for an electronic system:

$$
\frac{V_{\text {rec }}(t)}{\sqrt{50 \Omega}}=h_{N}(t) \circ \frac{E_{\text {inc }}(t)}{\sqrt{377 \Omega}}
$$

where $V_{\text {rec }}(t)$ is the received voltage at the output of an antenna, $E_{\text {inc }}(t)$ is the incident electric field at the antenna position, and the $\circ$ symbol stands for the convolution in time. A way to measure or simulate the antenna response is to set two of the same antennas in front of each other, excite one of them with a short pulse, and deduce the antenna response from the output of the second one (see scheme in fig. 4). We simulated this setup with Remcom XFDTD software [16], a solver of Maxwell's equation in time domain. A Gaussian pulse of around $200 \mathrm{ps}$ width is set as input to the emitting antenna and the output of the second antenna is recorded. Knowing the input voltage $V_{s r c}$ and the received voltage $V_{\text {rec }}$, one can extract the antenna response in frequency domain:

$$
\tilde{h}_{N}(f)=\sqrt{\frac{r c}{i f} \times \frac{\tilde{V}_{r e c}(f)}{\tilde{V}_{s r c}(f)} \times \frac{1}{e^{-\frac{2 \pi f r}{c}}}}
$$

where $r$ the distance between emitter and receiver, $c$ the speed of light and $i^{2}=-1$ and the tild sign means that the quantity under it is taken in the frequency space. The time response is then found going back in the time space. An example of the time response at the antenna boresight and the corresponding gain magnitude are shown in figure 4.

\subsection{Validation}

In order to validate our detector understanding, we also carried out calibration runs when the beam was off. With a fast pulse generator (Avtech AVNH-1-C) we produced a square pulse of $2.5 \mathrm{~ns}$ emitted with a commercial bicone antenna and we measured the electric field with the experimental setup ( $\mathrm{cf}$ fig. 5 left). From the input pulse we simulated the full chain of emission and reception. The time domain response of the bicone was simulated with the procedure described 

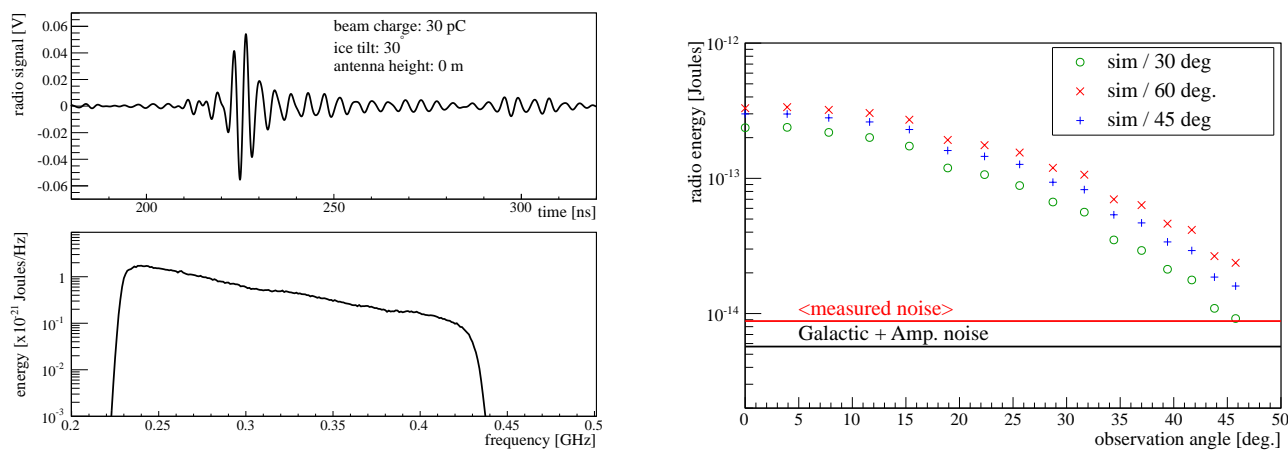

Figure 6: Left: expected waveform from Askaryan radiation after the full detector simulation. Right: Expected integrated power as a function of the angle between the ice target and the antenna ( 0 degree is defined at the horizontal).

above. A waveform comparison of measured and simulated waveforms is shown in fig. 5 (right). The main features are well reproduced and the total error on the time integrated power is less than $15 \%$.

\section{Full chain simulation}

We combine the previous studies to obtain the expected signal from the ARAcalTA experiment. A typical waveform and the corresponding spectrum are shown in fig. 6 left. The main peak extends over $40 \mathrm{~ns}$ and its amplitude reaches tens of $\mathrm{mV}$ which makes it detectable with an oscilloscope. The main features of the waveform are in fact driven by the amplifier and filter response.

The set of configurations (antenna heights/ice tilts) were simulated and the expected integrated power is shown in fig. 6 (right) as a function of the observation angle (see the caption). For all the configurations the expected power is found to be larger than the thermal background noise (including Galactic noise and amplifier noise) or the actual noise measured on site.

\section{Conclusion and discussion}

We have implemented a complete chain of simulation to estimate the signal in ARAcalTA experiment from the electric field emission to the antenna and detector response. The detector simulation was checked with a dedicated experiment. The estimated signal for Askaryan radiation is found to be larger than the thermal noise by a factor 2 for nearly every experimental configurations. One should note that the current simulated electric field accounts only for the Askaryan like radiation, i.e. issued from the individual energy losses of charged particles. We haven't simulated here any other emission aside from Askaryan radiation. Two potential backgrounds exist: transition radiation, that will occur when the electron beam penetrates the ice, and the sudden birth radiation, that will occur from the sudden appearance of the beam as it exits the pipe. These emissions may have an important contribution and their simulation are part of our current work. 


\section{References}

[1] ARA Collaboration, Astroparticle Physics 35 (2012) 457-477

[2] G.A. Askaryan, Sov. Phys. JETP 14 (1962) 441

[3] D. Saltzberg et al., Phys. Rev. Lett. 86 (2001) 2802

[4] The Pierre Auger Collaboration, Phys. Rev. D89 (2014) 052002

[5] P.W. Gorham et al., Phys. Rev. D72 (2005) 023002

[6] P.W. Gorham et al., Phys. Rev. Lett. 99 (2007) 171101

[7] K. Mase et al., PoS(ICRC2015)1136, these proceedings

[8] T. Shibata et al. Nucl.Instrum.Meth. A597 (2008) 61-66

[9] J. Alvarez-Muñiz et al.,Phys. Rev. D84 (2014) 012

[10] S. Agostinelli et al. for the GEANT4 Collaboration, Nucl.Instrum.Meth. A506 (2003) 250-303

[11] E. Zas et al. Phys.Rev. D45, (1992) 362-376

[12] C.W. James et al Phys. Rev. E84 (2011) 056602

[13] K. de Vries, arXiv:1503.02808

[14] J. Alvarez-Muñiz et al.,Astroparticle Physics 35 (2012) 325-341

[15] E. Farr, http://www.farr-research.com/Papers/ssn555.pdf.

[16] http://www.remcom.com/xf7 